\title{
T2K Near Detector Upgrades and Plans for T2HK
}

\author{
Thorsten Lux ${ }^{* \dagger}$ \\ Institut de Física d'Altes Energies (IFAE) - Barcelona Institute of Science and Technology \\ (BIST), Bellaterra (Barcelona), Spain \\ E-mail: Thorsten.Luxeifae.es
}

$\mathrm{T} 2 \mathrm{~K}$ is a long baseline neutrino experiment situated in Japan. T2K has established CP violation in the lepton sector on a $2 \sigma$ confidence level. To improve this result to better than $3 \sigma$ confidence level, the T2K collaboration proposes to continue the data taking beyond 2021 and to upgrade the neutrino beam and the T2K near detector. The detector upgrade consists in the installation of 3 new subdetectors in the existing near detector: A Super Fine Grain Detector acting as neutrino target, 2 high angle Time Projection Chambers and a Time of Flight detector. In this article the R\&D efforts of the T2K ND Upgrade Working Group towards these new subdetectors are summarized. Also an outlook on the plans beyond 2026 is given.

NuFACT2018

12th to 18th of August, 2018

Blacksburg, VA, USA

${ }^{*}$ Speaker.
${ }^{\dagger}$ For the T2K ND Upgrade Working Group 


\section{Introduction}

The Tokai to Kamiokande (T2K) is a long baseline neutrino oscillation experiment situated in Japan [1]. An intense beam of muon neutrinos is produced at the accelerator complex J-PARC (Tokai)( Fig. 1a). The statistics accumulated since 2010 has allowed to establish charge-parity (CP) violation in the lepton sector by comparing $v_{\mu} \rightarrow v_{e}$ and $\overline{v_{\mu}} \rightarrow \overline{v_{e}}$ oscillation probabilities at $2 \sigma$ confidence level for both mass hierarchies [2].

To achieve confidence levels above $3 \sigma$ until 2026 a continuation of the physics run beyond 2021, T2K-II, is proposed by the collaboration. The T2K-II proposal consists of an upgrade of the beam power from $485 \mathrm{~kW}$ to $1.3 \mathrm{MW}$ [3] and an upgrade of the current Near Detector (ND) complex [4]. T2K-II will be followed by the construction of a new far detector, HyperKamiokande (HK) [5] and an additional intermediate detector at $1 \mathrm{~km}$ based on a Water Cherenkov detector.

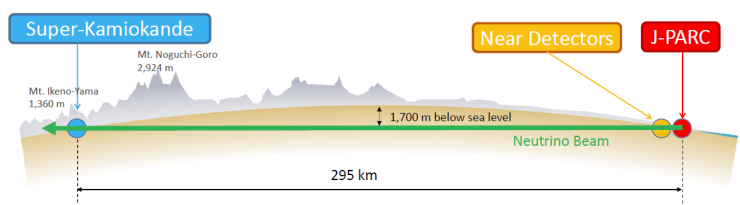

(a)

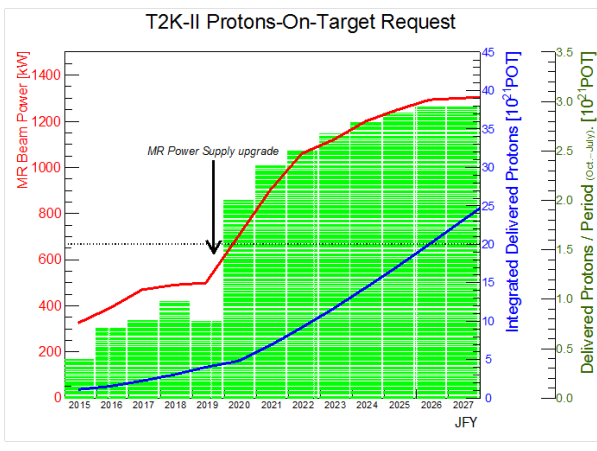

(b)

Figure 1: a) The schematic view of the T2K experiment and the location of its two detectors, the ND at J-PARC in Tokai and in a distance of $295 \mathrm{~km}$ the far detector SuperKamiokande. b) The expected beam power evolution over the years.

\section{T2K-II Near Detector Concept}

The current ND280 detector consists of 2 detector parts, the P0D, optimized for neutral current events, and a tracker composed of 3 TPCs and 2 Fine Grain Detectors (FGD) for the detection of charged current interactions [1]. Both of these detector parts are surrounded by an Electromagnetic CALorimeter (ECAL) and Side Muon Range Detectors (SMRD). For the upgrade it is foreseen to remove the POD and to replace it by a new tracker system consisting of a Super Fine Grain Detector (SuperFGD), 2 horizontal TPCs and a Time-of-Flight (TOF) detector. The upgraded detector will not only have twice the target mass for CCQE interactions but will improve especially the detection efficiency for leptons leaving the detector under a large angle.

\subsection{SuperFGD}

The SuperFGD is the core element of the upgraded ND280. It serves 2 purposes: it is the target for the neutrino interactions and at the same time a fully active detector allowing to reconstruct 
the tracks leaving the interaction point. The SuperFGD follows a novel detector approach for fine grained scintillator trackers [6]. In the current T2K FGDs (FGDYX) scintillator bars with wavelength shifter (WLS) fibers are grouped in orthogonal layers, each layer providing one transversal coordinate. The SuperFGD will provide directly a full 3D reconstruction of the event. This will be achieved by using optically isolated scintillator cubes, each $1 \times 1 \times 1 \mathrm{~cm}^{3}$, instead of bars. Three orthogonal WLS fibers ( Y-11 (200) from Kuraray), instrumented on one end by Multi-Pixel Photon Counters (MPPC), will go through each of the cubes. The dimensions of the SuperFGD will be $192(\mathrm{~W}) \times 192(\mathrm{~L}) \times 56(\mathrm{H}) \mathrm{cm}^{3}$ for a total mass of about 2 tons. The average light yield was measured to 41 p.e. for a MIP per fiber and the average optical cross talk was found to be $3.7 \%$.

The performance of the SuperFGD approach was studied with simulations and indicate that this detector concept outperforms the classical FGD approach (Fig. 2).

A prototype consisting of 9216 cubes and 1728 fibers $\left(8 \times 24 \times 48 \mathrm{~cm}^{3}\right)$ has been characterized in a
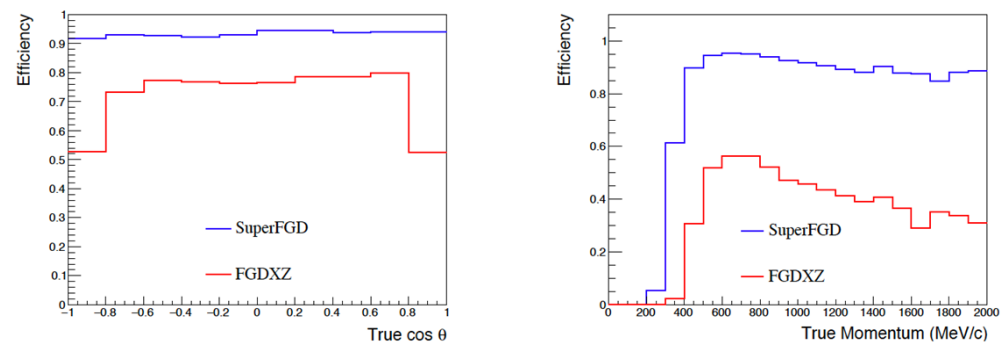

Figure 2: Left: The detection efficiency for muons in function of the true $\operatorname{cosine} \theta$. Right: The detection efficiency for protons in function of their true momentum. In both cases the novel approach of the SuperFGD is superior to the classical 2D FGD.

test beam. A detailed analysis of the test beam data is ongoing.

\subsection{High Angle TPC}

For the upgraded ND280 detector 2 new TPCs, the so-called high angle (HA) TPCs, will be constructed. TPCs, as low mass detectors, are especially suitable since the particles emitted in these directions are of low momentum of a few hundred MeV/c. To maximize the number of particles reaching the sensitive volumes, the walls of the field cage will be of composite material with a material budget of only $1.7 \%$ radiation length. While for the current TPCs bulk Micromegas (MM) [7] are used for the readout, resistive MM will be installed in the HA-TPCs. The pads of resistive MM are covered by 2 layers, one insulating and one of resistive material. The charge deposited by the avalanche on the resistive layer spreads naturally with time over several pads allowing to reach excellent spatial resolution even for small drift distances. The optimal resistivity is $0.4 \mathrm{MOhm} / \mathrm{square}$ which will yield a spread of $2.6 \mathrm{~mm}$. A first prototype was characterized in summer 2018 at a test beam at CERN. The pad size was $9.8 \times 7 \mathrm{~mm}^{2}$ and the resistivity was 2.5 $\mathrm{MOhm} / \mathrm{square}$. The point resolution was measured with this prototype for various particle types for 3 drift distances. The preliminary results indicate point resolutions between $350 \mu \mathrm{m}$ for $10 \mathrm{~cm}$ drift and $550 \mu \mathrm{m}$ for $80 \mathrm{~cm}$ drift. 


\subsection{Time-of-Flight Detector}

The Time-of-Flight (TOF) detector [8] aims at providing a measurement of the direction of the particle tracks observed within the detector. The time is measured between the TOF scintillator (EJ-200) panels on one hand and the SuperFGD on the other hand. To separate tracks entering the detector from the outside, considered background, to the ones starting within the SuperFGD and leaving the detector a time resolution of $500 \mathrm{ps}$ is required. A large prototype was tested successfully in a test beam proving a time resolution of $70 \mathrm{ps}$ using electronics with better time resolution as in the final detector.

\subsection{Expected Performance}

The expected performance of the upgraded ND280 detector was studied with simulation studies. The result is shown in Fig. 3. For comparison the detector efficiency for muons as function of $\cos \theta$ is also shown for the current ND280. As expected the efficiency is significantly improved for high angle tracks, while the current ND280 is optimized for forward tracks.

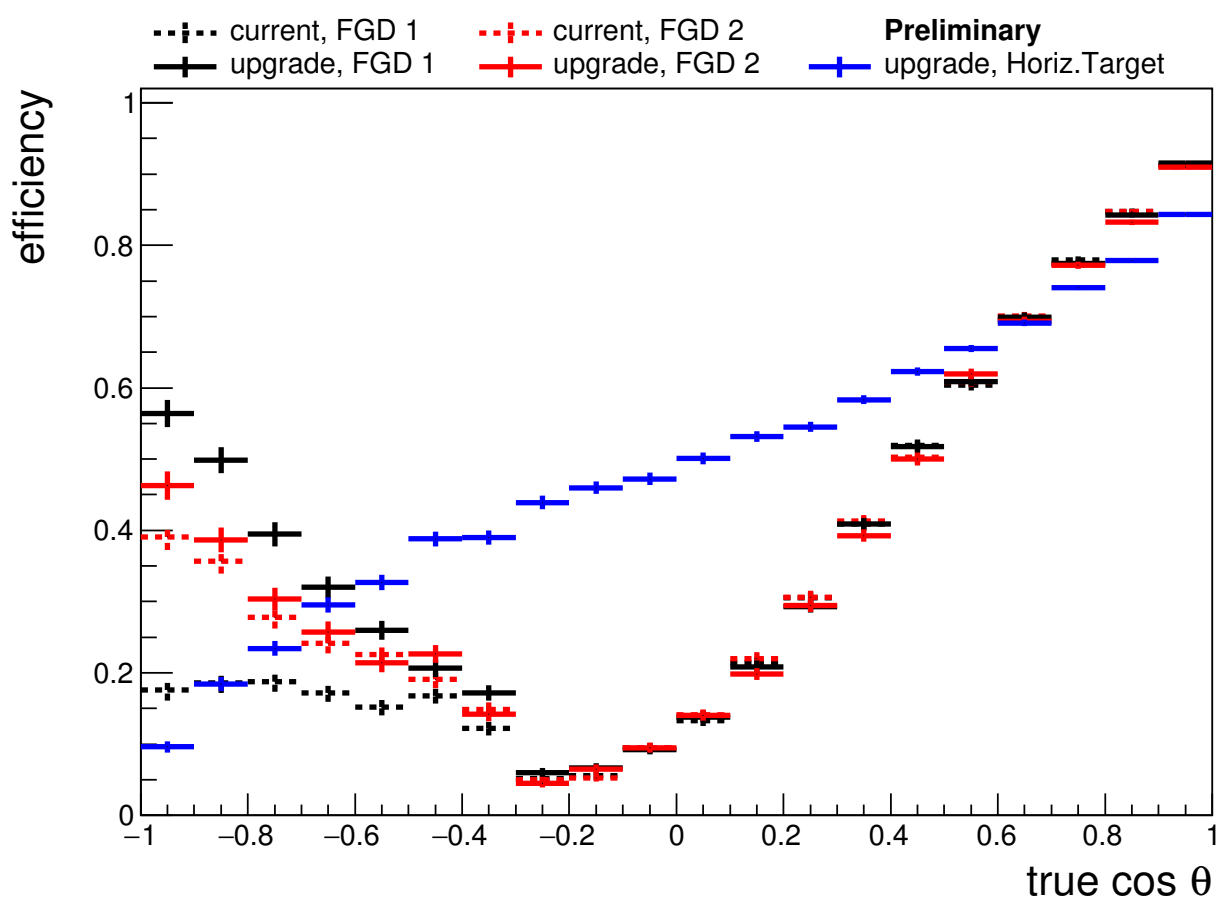

Figure 3: Muon detection efficiency as expected from simulations for the upgraded ND280. The dashed black and red markers show the muon detection efficiency for the current FGDs with a high inefficiency for muons leaving the detector under high angles. The solid black and red markers show the improved performance in the backward direction due to the implementation of the TOF panels. The solid blue markers show the detection efficiency of the upgraded detector with the SuperFGD as horizontal targed and HA-TPCs above and below the SuperFGD.

\section{Beyond T2K-II Upgrades}

While the proposed upgrade for the ND280 detector will improve the systematic error for the 
oscillation results from currently about $6 \%$ to $4 \%$, this is not enough to fulfill the requirement for HyperKamiokande (HK) of 3\%. For this reason an intermediate Water Cherenkov detector is proposed, to be installed $1 \mathrm{~km}$ from the target. To achieve a quasi mono-energetic neutrino beam, this detector will be moved between an Off-axis angle of 1 to 4 degrees. A detailed description of this project can be found in [9].

\section{Summary and Outlook}

T2K takes successfully data since 2009 which has led to an observation of CP violation on a $2 \sigma$ confidence level. To improve this result further, the T2K collaboration proposes to continue the data taking beyond 2021. For this T2K-II phase an upgrade of the beam power and the near detector is foreseen. An extensive $R \& D$ program is currently being carried including various test beam campaigns to characterize the prototypes of the different subdetectors. Also simulation studies were performed indicating a significantly better performance for the upgraded detector, especially for particles leaving the detector at high angles. At the same time a R\&D project studies the feasibility of an intermediate Water Cherenkov detector to reduce the systematic error even further. The new intermediate detector and also the new far detector, Hyper-Kamiokande, with a 10 times larger fiducial mass will start data taking 2026.

\section{Acknowledgments}

The author has received funding from the European Unions H2020 Grant No. RISEGA644294JENNIFER and from the Spanish Ministerio de Economia y Competitividad (SEIDI-MINECO) under Grants no. FPA2016-77347-C2-2-P and SEV-2016-0588 for the T2K ND280 Upgrade project. The author also would like to thank the CERN Neutrino Platform (CENF) for their support during the test beam campaigns of the prototypes.

\section{References}

[1] K. Abe, N. Abgrall, H. Aihara, Y. Ajima, J. Albert, D. Allan et al., The t2k experiment, Nuclear Instruments and Methods in Physics Research Section A: Accelerators, Spectrometers, Detectors and Associated Equipment $\mathbf{6 5 9}$ (2011) 106 - 135.

[2] T2K collaboration, K. Abe et al., Search for CP Violation in Neutrino and Antineutrino Oscillations by the T2K Experiment with $2.2 \times 10^{21}$ Protons on Target, Phys. Rev. Lett. 121 (2018) 171802, [1807.07891].

[3] M. Friend, J-parc accelerator and neutrino beamline upgrade programme, Journal of Physics: Conference Series 888 (2017) 012042.

[4] T2K Collaboration collaboration, A. Blondel, M. Yokoyama and M. Zito, The T2K-ND280 upgrade proposal, Tech. Rep. CERN-SPSC-2018-001. SPSC-P-357, CERN, Geneva, Jan, 2018.

[5] HyPeR-KAmioKANDE collaboration, Hyper-Kamiokande Design Report, .

[6] A. Blondel, F. Cadoux, S. Fedotov, M. Khabibullin, A. Khotjantsev, A. Korzenev et al., A fully-active fine-grained detector with three readout views, Journal of Instrumentation 13 (2018) P02006. 
[7] J. Bouchez, D. Burke, C. Cavata, P. Colas, X. D. L. Broise, A. Delbart et al., Bulk micromegas detectors for large tpc applications, Nuclear Instruments and Methods in Physics Research Section A: Accelerators, Spectrometers, Detectors and Associated Equipment 574 (2007) 425 - 432.

[8] C. Betancourt, A. Blondel, R. Brundler, A. DÃd'twyler, Y. Favre, D. Gascon et al., Application of large area sipms for the readout of a plastic scintillator based timing detector, Journal of Instrumentation 12 (2017) P11023.

[9] J-PARC E61 collaboration, E. Drakopoulou, An intermediate water Cherenkov detector for the Hyper-Kamiokande experiment: overview and status, PoS ICRC2017 (2018) 1021. 